\title{
Boost Trajectory Design for RBCC Engine
}

\author{
Tan Zhichen \\ College of Astronautics \\ Northwestern Polytechnical University \\ Xi’an, China \\ hellomyth@163.com
}

\author{
Zhang Ke \\ College of Astronautics \\ Northwestern Polytechnical University \\ Xi’an, China \\ zhangke@nwpu.edu.cn
}

\begin{abstract}
With the development of the next generation of reusable aerospace vehicle, the technique of the Rocket Based Combined Cycle (RBCC) engine becomes increasingly significant and valuable. However, the performance of RBCC engine is coupled closely with the process of trajectory design. A model based on Radan Pseudospectral Optimal Method aiming at designing the boost trajectory is proposed in this paper. Two typical trajectories differing in variable and constant dynamic pressure constraints are simulated and compared. The constant dynamic pressure trajectory is designed simply with an acceptable optimal result. The feedback linearization algorithm to track the referent trajectory is analyzed and the simulated result shows its efficiency.
\end{abstract}

Keywords-Reentry; Hypersonic; Trajectory Optimization; RBCC; Gauss Pseudospectral Method

\section{INTRODUCTION}

Advanced hypersonic airbreathing propulsion is of significant interest for possible application to the future because of its low cost and reusable space transportation. The Rocket Based Combined Cycle (RBCC) engine is a perfect choice for the dynamic systems. Astrox Corporation has been tasked by the Office of the Chief Scientist of the Air Force to study the space transportation systems, and RBCC engine was selected as one part of the dynamic systems[1].

The performance of the RBCC engine is coupled with the states of working. Therefore, boost trajectory design plays an important role in the nature of RBCC engine which supplies the dynamics for vehicle in boost phase. On one hand, Isp performance couples with the vehicle's Mach, angle of attack(AoA) and dynamic pressure. Isp nature can also lower fuel consumption. On the other hand, the dynamic pressure can influence the structure loading and the aerodynamic heating. High dynamic pressure may cause damage of the structure of the vehicle.

References 2-7 have carried out a lot of research on the RBCC engine working in boost phase. R.Odls et[8] used POST (Program to Optimize Simulated Trajectories) to simulate the constant dynamic pressure trajectory and analyzed three methods to guide the vehicle. Optimal mission performance and multivariable flight guidance for airbreathing launch vehicle has been analyzed in detail and the energy-state approach was employed[9]. However, some hypothesis was set up to solve the problem.

Radan Pseudospectral Optimal Method[10,11] is employed to design the RBCC engine boost trajectory, and the feedback linearization theory[12] is used to track the designed trajectory in this paper. This paper is organized as follows: First of all, boost dynamics is described. Second, the control variables are analyzed on the constant pressure condition. Two typical trajectories are simulated and compared based on optimal method at the third part. The tracking algorithm is analyzed at the forth part. The conclusion is presented at the last part.

\section{DYNAMICS IN BOOSTER PHASE}

To formulate boost trajectory design problem, a twodimension model in the pitch plane is applied in this paper and the point-mass dynamics of a vehicle in the inertial launch frame are described as follows:

$$
\begin{gathered}
m \dot{v}=P \cos \alpha-G \sin \theta-D \\
m v \dot{\theta}=P \sin \alpha-G \cos \theta+L \\
\dot{x}=v \cos \theta \\
\dot{y}=v \sin \theta \\
P=\dot{m} I s p
\end{gathered}
$$

Where $m$ is the mass of the launch vehicle, $v$ is the launch vehicle velocity, $\alpha$ is the AoA, $\theta$ is the flight path angle, $P$ is the propulsion, $G$ is the gravity, $D$ is the drag force, $L$ is the lift force, $x$ and $y$ represent the position of the vehicle center of mass in the inertial launch frame, and $\dot{m}$ is the fuel consumption per second.

\section{A. Constant pressure}

To obtain the constant pressure condition in boost phase, a rigorous constraint condition about velocity and height of the vehicle should be satisfied. Recur to the atmosphere density equation

$$
\rho=\rho_{0} e^{(-y / \beta)}
$$

Where $\rho_{0}$ is the density value at sea level and $\beta$ is the nominal height parameter.

Dynamic pressure can be written as the following equation:

$$
q=0.5 \rho_{0} v^{2} e^{(-y / \beta)}
$$

The condition that the value of dynamic pressure kept constant with time increasing is assumed. Thus, the equation of the mutative rate of dynamic pressure is given as

$$
\frac{d q}{d t}=0
$$

According to formulas (1) and (4), formula (8) can be written as

$$
P \cos \alpha=G \sin \theta+D+v^{2} \sin \theta / 2 \beta
$$

If formula (9) could be satisfied rigorously in boost phase, the dynamic pressure will be a constant value. With the variety of state parameters of the right part of formula(9), the sum of the three parts may change rapidly. On this condition, AoA should be adjusted. Because of the limits of the bounds of the AoA value and the mutative rate value, the thrust of the RBCC engine may be adjusted to insure the condition that the sum of the left part of 
formula (9) equals to the right part. Whereas, the thrust is the product of Isp and the consuming mass of fuel, and Isp of the fuel is coupled with AoA. AoA can be selected as the control variable parameter to obtain the constant dynamic pressure boost trajectory.

\section{OPTIMAL TRAJECTORY}

Two typical optimal booster trajectories differing in constant and various dynamic pressure constraints are simulated in this part. If the dynamic pressure curve was determined, the $\mathrm{h}-\mathrm{v}$ curve can be described. Thus, the trajectory design can be translated into the task of calculating guidance commands at each state to insure the velocity and height of the vehicle can be close to each referent state, and the object of the least fuel consumptions can be set to obtain a perfect performance.

The sonic speed is assumed as a constant value equals to $300 \mathrm{~m} / \mathrm{s}$. The initial and terminal states are depicted in Table I .

TABLE I. THE INITIAL AND TERMINAL STATES OF THE BOOSTER PHASE

\begin{tabular}{|c|c|c|c|c|c|}
\hline & Ma & $\mathbf{Y}$ (meter) & Mass(kg) & $\boldsymbol{X}$ (meter) & $\theta\left(^{0}\right)$ \\
\hline $\begin{array}{c}\text { Initial } \\
\text { State }\end{array}$ & 4 & 20000 & 110000 & 0 & \\
\hline $\begin{array}{c}\text { Terminal } \\
\text { State }\end{array}$ & 10 & 31920 & & & \\
\hline
\end{tabular}

To simulate the dynamic performance of the movement of AoA, on one hand, the dynamic pressure is assumed to be constant and the magnitude is determined by the initial boost trajectory state parameters, the mutative rate of $\operatorname{AoA}(\dot{\alpha})$ is selected as the control parameter. On the other hand, if the dynamic pressure is variable, thrust and the mutative rate of $\operatorname{AoA}(\dot{\alpha})$ were selected as the control parameters. The constraints are depicted in Table $\mathbb{I}$.

TABLE II. THE CONSTRAINTS OF THE OPTIMAL PROCESS

\begin{tabular}{|c|c|c|c|c|c|}
\hline$\alpha\left({ }^{0}\right)$ & $\dot{\alpha}\left({ }^{0} / s\right)$ & $\theta\left(^{0}\right)$ & $y($ meter $)$ & $v(\mathbf{m} / \mathbf{s})$ & $p(\mathbf{N})$ \\
\hline$[1,8]$ & {$[-2,2]$} & {$[0,10]$} & $\begin{array}{c}{[20000,} \\
40000]\end{array}$ & $\begin{array}{c}{[1200,} \\
3000]\end{array}$ & {$\left[20 m_{\text {ini }}, 50 m_{\text {ini }}\right]$} \\
\hline
\end{tabular}

Assuming that Condition 1 is the symbol of the condition of the variable dynamic pressure and condition 2 is the symbol of condition of the constant dynamic pressure, and the optimal results based on Radan Pseudospectral Optimal Method are depicted from figure 1 to figure 5 .

From figure 1 to figure 4 , the conclusions that the total fuel consumptions of the condition 1 is a little less than the condition 2, and the bound of the dynamic pressure of condition 1 is much more acute than condition 1 can be obtained. The magnitude of the object of the condition 2 is close to the result of the condition 1 , and the dynamic pressure of the condition 2 is more available to protect the structure of the vehicle than the condition 1 . Therefore, the condition of the constant dynamic pressure can be set to the constraint in boost phase design process. Figure 5 shows that the process of condition 1 deals with the magnitude of the velocity and height increasing problem is more logically.

\section{PATH TRACKING ALGORITHM}

A path tracking algorithm based on feedback linearization is proposed in this part. As known that it is hard to set the optimal trajectory in the boost phase on time. Therefore, tracking the optimal trajectory designed previously is an efficient way to lower the fuel consumption.

The referent flight path angle mutative rate can be obtained from the designed trajectory at each time node, and the additional value can be obtained by feedback linearization model. The commanded flight path angle mutative rate is given by:

$$
\begin{gathered}
\dot{\theta}_{\text {com }}=\dot{\theta}_{r e f}+\Delta \dot{\theta} \\
\Delta \dot{\theta}=\xi \omega_{n}\left(\dot{h}_{r e f}-\dot{h}\right)+\omega_{n}{ }^{2}\left(h_{r e f}-h\right)
\end{gathered}
$$

Where ()$_{\text {ref }}$ is the referent state, $\xi$ is the damping ration and $\omega_{n}$ is the natural frequency.

Obviously, the value of the command AoA can be calculated by

$$
m v \dot{\theta}_{\text {com }} \approx \dot{m} I s p(\alpha, M a, h) \alpha+q S_{r e f} C_{L}(\alpha, M a)+m g \sin \theta
$$

Where $C_{L}(\alpha, M a)$ is adapted as a quadratic function about $\alpha$ at each state, and Isp is also adapted as a linear function about $\alpha$ at each state.

Mass specific energy $\mathrm{E}$ is employed as a state variable in pa place of velocity $\mathrm{v}$, where

$$
E=\frac{1}{2} v^{2}+g y
$$

If the states were defined each time, the added $\mathrm{E}$ obtained by the designed trajectory is given by

$$
\Delta E=E_{1}-E_{0}
$$

$E_{0}$ is the current specific energy and $E_{1}$ is the specific forecast energy.

To obtain the fuel consumptions each time and simplify the calculation process, the fuel consumption is given by

$$
\dot{m}=\left(m \Delta E / v+q S_{r e f} C_{D}(\alpha, M a)\right) / \operatorname{Isp}(\alpha, M a, h)
$$

Using the feedback linearization theory to track the path of the condition 2, the simulated results are given by

This feedback linearization tracking algorithm proposed in this part can guide the vehicle to track the referent trajectory efficiently. The total fuel consumption of the tracking algorithm is about $135 \mathrm{~kg}$ more than the designed condition.

\section{CONCLUSION}

The optimal boost trajectory design for RBCC engine can be predigested on the condition of a constant dynamic pressure, the optimal parameter can be selected so that no other value of and the value of thrust can be calculated by equivalent formula in the optimal process. Thus, the dimensions of the optimal parameters can be decreased and the efficiency of optimal process can increased. Even though the fuel consumption is not the optimal, one of the variable dynamic pressure constraint conditions, the sum of the fuel consumption can be close to the best one and the results are acceptable. Using the feedback linearization theory can guide the vehicle to track the referent trajectory 
efficiently, and the additional fuel consumption is a little more than the optimal result. All in all, the algorithm proposed in this paper may be helpful to future generation transportation vehicle trajectory design.

\section{REFERENCES}

[1] Ajay P. Kothari,John W. Livingston, Christopher Tarpley,etc., A Reusable, Rocket and Air-breathing Combined Cycle Hypersonic Vehicle Design for Access-to-Space[R], AIAA 2010-8905

[2] Liu Yang,He Guoqiang, Liu Peijing,et al., Present situation and progress of investigation on rocket based combined cycle(RBCC) propulsion system[J], Journal of Solid Rocket Technology, 2009, 32(3):288-293

[3] David A Young, Timothy Kokan. Lazarus: a SSTO hypersonic vehicle concept utilizing RBCC and HEDM propulsion technologies[R]. AIAA 2006-8099

[4] Kevin W. Flaherty, Katherine .M Andrews, Glenn W. Liston, Operability benefits of airbreathing hypersonic propulsion for flexible access to space[J]. Journal of Spacecraft and Rockets, 2010, 47(2): 3-4.

[5] Lu Xiang, He Guoqiang, Liu Peijin, Ascent Trajectory Design Method for RBCC powered Vehicle[J], ACTA AERONAU TICA, 2010,31(7):1331-1337

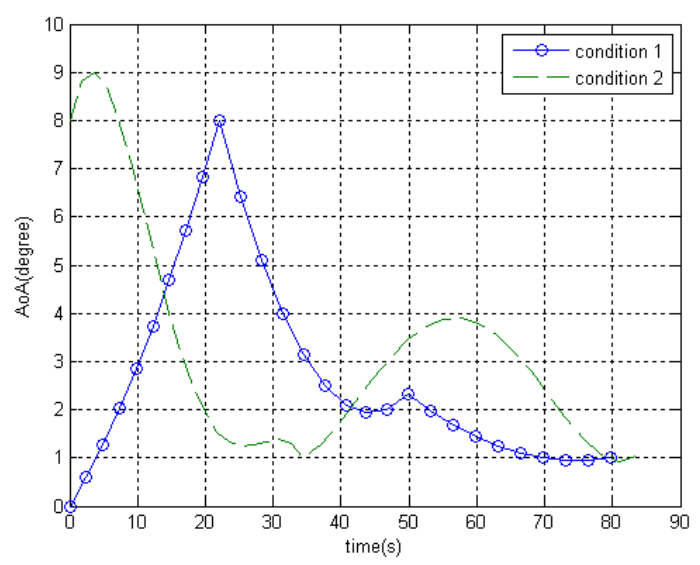

Figure 1 AoA profiles

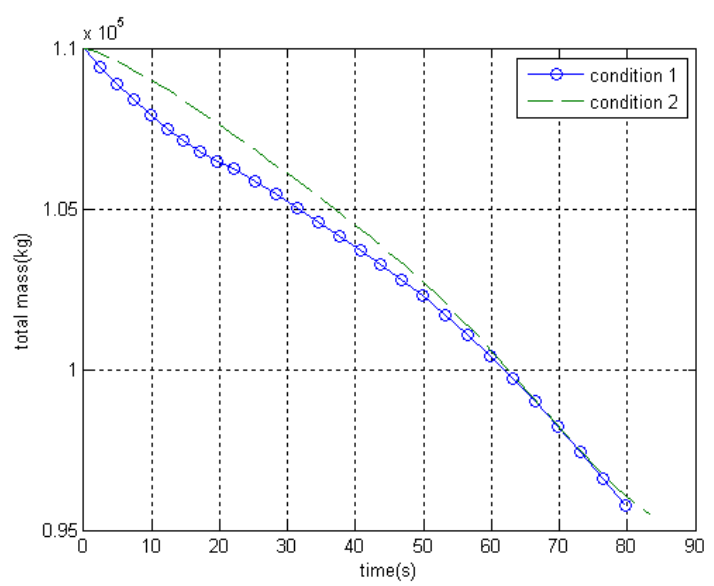

Figure 3 Total mass curves
[6] XUE Rui, HU Chun-bo, LV Xiang, et al., RBCC constant dynamic pressure booster trajectory design and propellant mass flowrate analysis for TSTO transportation system, Journal of Solid Rocket Technology,http://www.cnki.net/kcms/detail/61.1176.V.20121217. 0842.015.html, 2012.

[7] GONG Chun-lin, HAN Lu, Optimization of ascent trajectory for RBCC-powered RLV[J], Journal of Solid Rocket Technology, 2012,35(3):290-295.

[8] Olds J. R., Budianto I. A., Constant dynamic pressure trajectory simulation with POST[R], AIAA-1998-302, 1998.

[9] David K.Schmidt. Optimum Mission Performance and Multivariable Flight Guidance for Airbreathing Launch Vehicles[J], Journal of Guidance, Control, and Dynamics, 1997,20(6):11571164.

[10] Garg, D., Patterson, M. A., Hager,W.W., Rao, A. V., Benson, D. A., and Huntington, G. T., A Unified Framework for the Numerical Solution of Optimal Control Problems Using Pseudospectral Methods,Automatica, 2010,46(11): 1843-1851.

[11] Garg,D.,Hager,W.W.and Rao,A.V., Preudospectral Methods for solving Infinite-Horizon Optimal Control Problems, Automatica, PublishedOnlineMarch2010,DOI:10.1016j.automatica.2011.01.085

[12] J.E.Corban,A.J.Calise, and G.A.Flandro. Rapid Near-Optimal Aerospace Plane Trajectory Genetation and Guidance[J], Guidance,1991,14(6):1187-1188

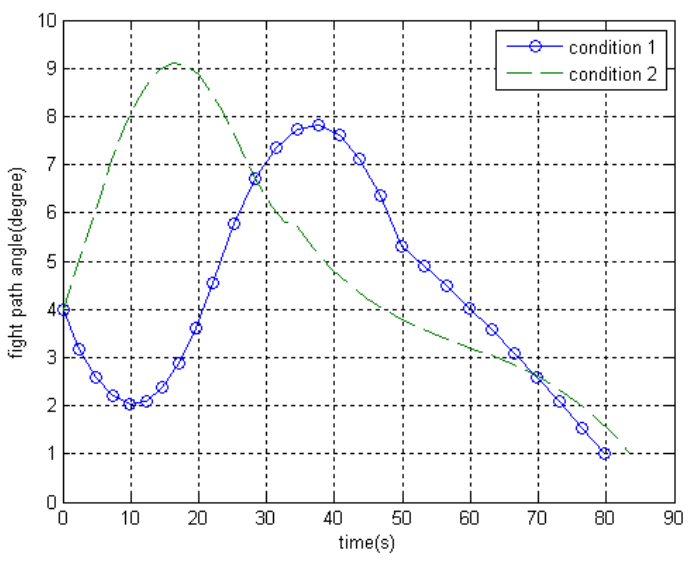

Figure 2 Flight path angle profiles

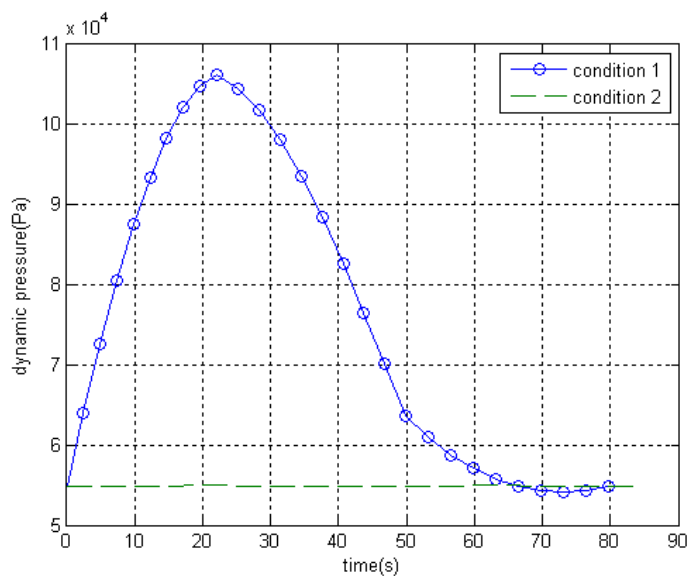

Figure 4 Dynamic pressure curves 


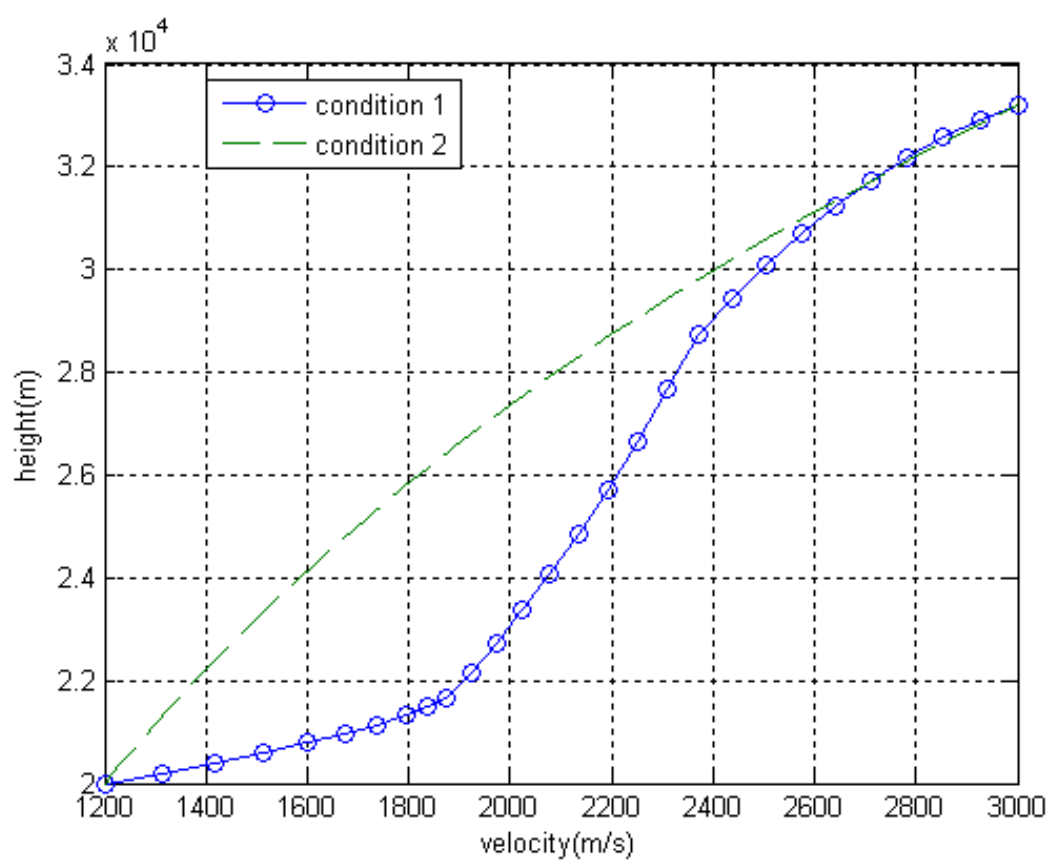

Figure 5 Height vs. velocity curves

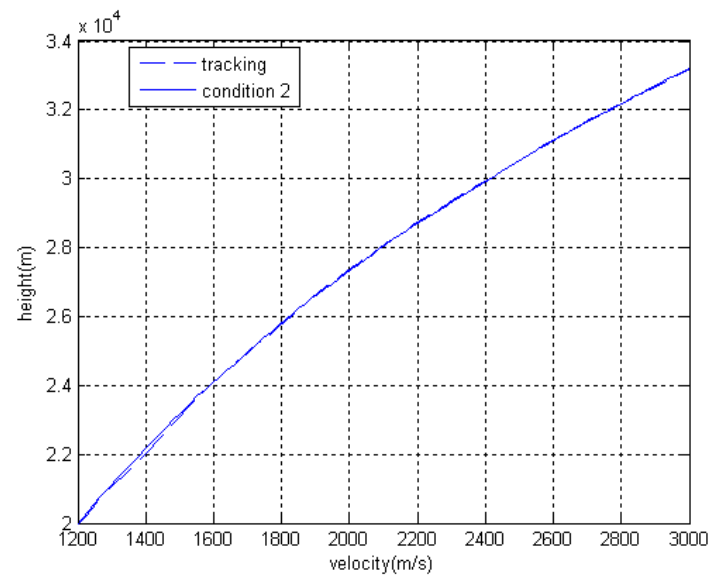

Figure 6 Height vs. velocity curve

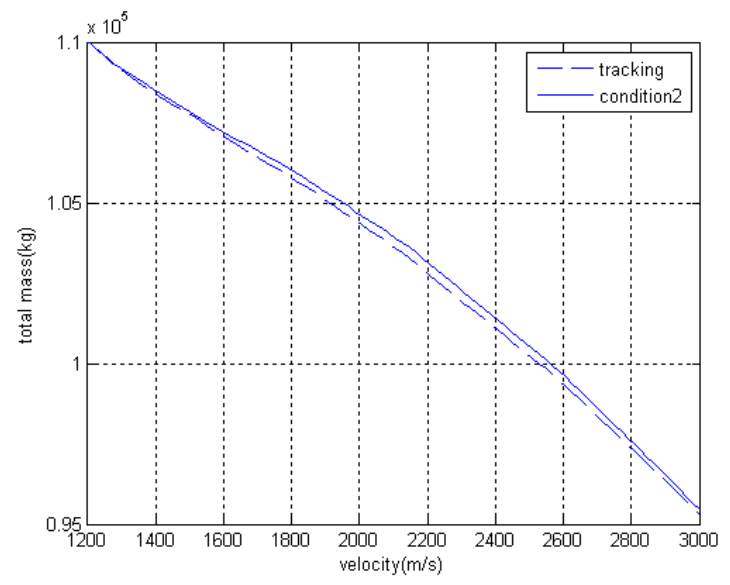

Figure 7 Total mass vs. velocity curve 